\title{
万古霉素的尘世之旅
}

\author{
钱璞凡†，吕萍* \\ 浙江大学化学系, 杭州 310027
}

摘要: 作为糖胝类抗生素家族中发现的首个分子, 万古雱素长久以来都被认为是多重耐药革兰氏阳性菌的最后一道 防线。本文利用几个叙述视角不一的短故事, 串起了万古霖素发现和发展的全过程, 通俗易懂地介绍了万古霸素, 并介绍了一些与万古霰素相关的生物化学和有机化学的概念。

关键词: 万古霉素; 革兰氏阳性菌; 放线菌; 糖肽类抗生素

中图分类号: G64; O6

\section{The Journey of Vancomycin in the World}

\author{
Pufan Qian ${ }^{\dagger}$, Ping Lü * \\ Department of Chemistry, Zhejiang University, Hangzhou 310027, China.
}

\begin{abstract}
As the first molecule found in glycopeptide antibiotic family, vancomycin has been considered as the last line of defense for multidrug-resistant Gram-positive bacteria for a long time. In this paper, we utilized several short stories from different narrative perspectives to summarize the whole process of discovery and development of vancomycin. Furthermore, we also introduced some biochemical and organic concepts related to vancomycin at the same time.
\end{abstract}

Key Words: Vancomycin; Gram-positive bacteria; Actinomycetes; Glycopeptide antibiotics

“啦啦啦啦, 啦啦啦, 我是破坏的小行家……”黄色葡萄球菌小黄正在他宿主小高的血浆中边 哼着小曲边搞破坏, 东拆一块砖, 西敲一片瓦, 还对周围环境中充斥着的青霉素熟视无睹。原来, 这 个细菌是一个技艺高超的青霉素耐药菌, 哪怕是青霉素类抗生素中最强势的对甲氧苯青霉素也拿他 束手无策 ${ }^{[1]}$ 。小黄一路如入无人之境, 眼看着就要突入血液-脑脊液屏障 ${ }^{[2]}$, 进入大脑驰骋了。正当 小黄神游天外的时候, 便迎面撞上了一个分子, 莫名的危机感让这个小小的细胞试图闭上与外界交 换物质的嘴巴, 可还是没来得及。不过小黄安慰自己, 这个分子虽然味道和以前不小心吃到的那些 青霉素类抗生素不尽相同 ${ }^{[3]}$, 但他可是无所不能的耐药菌。

可这次他错了。小黄在血管的转弯处撞了一下血管壁, 把细胞壁上的肽聚糖 ${ }^{[4,5}$ 撞落了一些, 他 这才想起来自己刚刚太贪吃, 身子长大了不少, 细胞壁也需要更新一次了。于是他启动了肽聚糖的 合成。可他却震惊地发现, 体内这一合成过程似乎遇到了一些阻力。仔细一看, 竟是刚刚吃进的那 个外来的分子, 正恰好接到了的肽聚糖链一端, 导致肽聚糖层间进一步的交联无法正常进行 ${ }^{[6]}$ 。这 可把小黄急坏了: 细胞壁无法更新, 他便失去了生长和繁殖的能力, 这对于一个单细胞原核生物而 言, 意味着生命将在几个小时的停滞后彻底终结。他有些不甘心, 便调动了细胞内所有的酶前往肽

收稿: 2021-06-21; 录用: 2021-07-14; 网络发表: 2021-08-09

†2018 级化学(求是科学班)本科生

“通讯作者, Email: pinglu@zju.edu.cn 
聚糖合成出错的位点, 试图将外来的这个奇怪分子解离下来, 继续合成肽聚糖。可不一会儿, 他便 颓然地发现, 自己体内的酶, 似乎都无法识别这个分子, 站在这个错误跟前束手无策。他绝望了。

没过几个小时, 小黄在绝望中死去了, 就像小高体内他那些同样进化出对甲氧苯青霉素耐药性 的同伴们一样。在濒死的时刻, 小黄依然想不通杀死自己的到底又是何方神圣。其实消灭他们的, 便 是多重耐药革兰氏阳性菌的天敌，万古霉素。

\section{1 初次相遇}

彼时, 东方拟无枝酸菌还只是一种无名放线菌, 小东便是出生在加里曼丹岛的一个 ${ }^{[7]}$ 。这天, 他 和往常一样, 懒洋洋地躺在泥土里感受太阳的温度。正当他快要睡着的时候, 突然感觉周围的泥土 开始了快速地上升, 吓得小东赶紧伸出鞭毛, 紧紧抓牢边上的泥土。经过一段漫长的下落后, 小东 突然觉得照在自己身上的光特别冷, 随后便失去了意识。

同小东一样，彼时的科恩菲尔德也同样寂寂无名。1953年，从哈佛大学博士毕业的他，正带领 着团队，从各类放线菌的代谢产物中为礼来公司寻找新的抗生素。在显微镜下，科恩看到了小东。 小东的样子和从前观察到的那些放线菌形态不同, 科恩心想, 这一定是一个稀有的菌种, 一定会有 其独特功能。想到这, 他便颇有干劲地开始从样本中分离小东。

和往常一样, 科恩分离了土壤样本中的全部菌种, 将他们分开在不同的培养基中, 尝试培养他 们的单菌落, 进而分离他们代谢产生的活性物种。在培养基的滋养下, 漂洋过海的小东开始了快速 繁殖, 成功培育成了一个单菌落, 培养基中也出现了一些小东族群的特异代谢产物。经过了更加漫 长的分离纯化过程后, 科恩发现, 在体外试验中, 这种无名细菌的培养液中分离得到的一种编号为 05865的活性成分, 对葡萄球菌有着很强的抑制作用, 甚至连对青霉素无感的金黄色葡萄球菌也有着 几乎相同的杀伤力。随后他们又进行了进一步的耐药性培养实验, 发现如果在含有活性成分05865的 培养基中培养金黄色葡萄球菌, 那怕是经过了多次传代培养, 葡萄球菌仍然没有被诱导出对于活性 成分 05865 的显著抗药性 ${ }^{[8,9]}$ 。

这一发现让科恩欣喜若狂。这简直是捡到宝了啊！近些年来, 由于这些耐药菌的横行, 对于那 些青霉素无法治愈的病人, 往往只能乞求奇迹出现, 可05865这种活性成分的出现竟然打破了这一 点。这简直是一个发大财的好机会呀! 在科恩菲尔德的建议下, 礼来公司将这种物质命名为万古霉 素(Vancomycin), 取自于英文单词Vanquish, 以彰显其对革兰阳性菌的 “绝对清除作用”。同时, 他们 也给漂洋过海的小东起了个名字: 东方拟无枝酸菌。自此, 默默无名的小东也终于有了他的名字 ${ }^{[10]}$ 。

现在唯一横在他们面前的问题就是纯度。对于批量生产, 礼来公司一点都不担心, 毕竟只要培 养基足够就行。可东方拟无枝酸菌的代谢产物实在太多, 很多又难以祛除, 在没有全部确定结构之 前就贸然开始临床试验，万一这些副产物中，有一些物质的毒性很大，那该怎么办？

这像一桶冷水又浇灭了科恩团队的喜悦。他们又开始了漫长的尝试, 先是尝试了用苦味酸进行 分离, 然后在此基础上对分离方法进一步改进, 但纯度一直都卡在 $80 \%$ 左右, 一直没能越过 $85 \%$ 的大 关。虽然体外实验都证明了万古霉素的高效性, 但这如果其中的杂质对其他器官有害, 可不就是对 病人不负责任? 如果确实能够找到危重病人, 愿意尝试新药, 那倒也是一大幸事。

\section{2 小试牛刀}

疻晨, 躺在病床上的爱德华被一阵疼痛疼醒。“哎, 吗啡的效果又过去了, 今天又注定是一个不 眠夜了。” 爱德华望着窗外的明月, 心中百感交集。一个月前, 因为脚部出现了一些小病灶, 需要手 术根除, 他便住进了这家医院。手术非常顺利, 医生说不会有复发的风险, 伤口不发生感染的话两 周就可以出院。可没想到命运就是捉摸不透, 第二天伤口便发生了细菌感染, 而且在青霉素的作用 下也丝毫没有溃退的趋势。有次医生来查房的时候他问起来, 医生犹豫半天, 才支支吾吾地告诉 他, 可能是青霉素耐药菌感染, 不过也鼓励爱德华不要放弃希望, 一定是有办法的。 
清晨的阳光从外面酒了进来, 新的一天又开始了。昨天早上医生来查房的时候通知他说, 如果 感染再扩大, 细菌随时可能进入血液循环系统, 感染很快会扩散到全身, 到时候真地就无力回天了。 事到如今, 截肢或许是最保险的办法。医生说, 现在还没有对抗耐药菌的特效药, 或许只能等待奇 迹出现。

正在爱德华胡思乱想之际, 病房的门被推开了。医生快步走进来: “爱德华! 我的朋友听说了你 的病症, 他告诉我说, 礼来公司研发了一款新药, 对于耐药菌有奇效, 目前已经进入了 II期临床试验 阶段。如果你愿意使用的话, 我便去问一问, 能不能给你申请一点试一试? ”

爱德华简直不敢相信自己的耳朵。好像自己有救了？“好的好的，谢谢医生！”

当晚, 礼来公司的员工便将万古霉素送了过来, 特别嘱咐说需要每天给药两次, 效果才比较好。 考虑到爱德华的情况特别危急, 医生决定每 8 小时给药 100 毫克的剂量 ${ }^{[11]}$, 用药一周。爱德华不会想 到, 第二天早上, 之前青霉素一直无能为力的感染竟好了大半。一个疗程还没结束, 感染便已全部 被祛除, 可以出院了 ${ }^{[12]}$ 。

\section{3 当头一棒}

就这样, 1958年, 由于万古霉素对于抗耐药菌感染的奇效, 礼来公司在未确定万古霉素结构的 基础上便将其匀匀上市了 ${ }^{[13]}$ 。刚上市的几年, 万古霉素确实因为其对于耐青霉素的革兰阳性菌都有 显著效果而受到医生和病人们的追捧, 但随后几年中发生的两个不大不小的事情, 却迫使人们又重 新冷静了下来。

第一个事情是一个小小的医疗事故 ${ }^{[14]}$ 。一家医院在治疗一位患有化脓性肌炎和骨髓炎的4岁男 孩时, 在使用万古霉素进行治疗时, 出现了严重的发热现象。由于万古霉素在此之前也有肾毒性的 相关报道, 因此医生选择了停用万古霉素, 改用了新药进行治疗。一停用万古霉素, 男孩持续的不

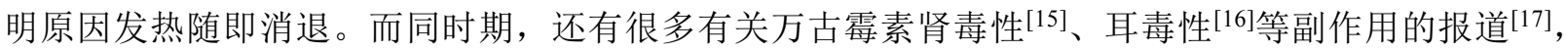
这使得万古霉素的安全性受到了一些质疑。

第二个事情出乎意料但又在情理之中。耐万古霉素的细菌出现了 ${ }^{[18]}$ 。在长久的临床治疗中, 万 古霉素一直被当做是感染的最后一道防线。如今, 这一道防线竟然破了, 那该怎么办?

这两个问题鞭策着化学家们对万古霉素的结构进行确定, 研究其致病机理, 进而开发出更多新 药, 才能解决抗药性这个问题。

\section{4 豁然开朗}

时间一晃而过，来到了1980年代。康斯坦斯·哈里斯正端坐在实验室的电脑前查阅有关万古霉素 结构确定的文献。他正在浏览谢尔德里克1978年在Nature 上发表的有关万古霉素结构鉴定的工作 ${ }^{[19]}$ 。 其实在之前, 对万古霉素结构的探索, 已经有了很多阶段性的成果。1965年, 马歇尔将万古霉素降 解, 尝试分析降解所得的产物, 初步确定了万古霉素的基本骨架结构, 迈出了关键一步 [20]。1977年, 威廉姆斯和卡尔曼借助核磁共振仪给万古霉素做了一个全身体检, 在氢核磁共振谱中分别观察到万 古霉素中 6 个肽键 $(-\mathrm{CONH}-)$ 和 1 个酰胺键 $\left(-\mathrm{CONH}_{2}\right)$ 的信号; 考虑到肽链中氮原子的鳌合配位能 力, 他们分析了不同半径的 $\mathrm{Eu}^{3+}$ 和 $\mathrm{Pr}^{3+}$ 离子与万古霉素结合形成的产物, 对于万古霉素的骨架结构给 出了更加具体的指导意见 ${ }^{[21]}$, 不过后来他们又对这一结构中芳环的构象进行了微小的修正 ${ }^{[22]}$ 。谢尔 德里克则在他们的基础上进一步发展, 用X射线从不同角度给万古霉素降解产物CDP-I的单晶照了许 多张照片, 将各个角度的图像叠加起来, 还原了CDP-I的分子结构, 并以此为基础, 结合其他降解产 物的结构, 提出了万古霉素的结构 $[19]$ 。

但谢尔德里克的CDP-I的结构中并不含有酰胺键 $\left(-\mathrm{CONH}_{2}\right)$ 。哈里斯在前期的工作后发现, 似乎 有更多证据支持威廉姆斯含有酰胺键 $\left(-\mathrm{CONH}_{2}\right)$ 的判断。他认为, 基于降解产物CDP-I结构推断出的 万古霉素异天冬酰胺残基独特结构实际可能是在万古霉素降解过程中重排造就的, 而这个残基的前 
体应该就是更常见的天冬酰胺。

但他一直苦于没能拿到实验证据。

“康斯坦斯, 又在想你那个万古霉素了? ”他的同事托马斯·哈里斯端着杯咖啡走过来问道。

“是啊, 还是没想到该怎么设计实验拿到关键证据。”康斯坦斯显得有点气馁。

“你呀, 就是又把问题复杂化了。既然你都有了猜想, 那就先把它解决了, 再想其他复杂的事 情。先针对你的猜想, 去查查有哪些反应是天冬酰胺所独有的, 哪些反应是异天冬酰胺残基独有的, 要是两者的反应性相互印证, 那结果不就显而易见了嘛。”

康斯坦斯眼前一亮, 当即开始设计实验。首先, 相比于仅碳原子端连有基团的酰胺键 $\left(-\mathrm{CONH}_{2}\right)$, 肽键 $(-\mathrm{CONH}-)$ 被碳原子和氮原子伸展出去的两条链固定在中间, 具有更大的位阻; 倘若使用乙硼 烷对这两种位阻不同的酰胺键进行还原, 由于肽键周围基团更丰富, 对于外来试剂的排斥更加明显, 导致了乙硼烷靠近时需要克服的阻力和能量要多一些、消耗的时间要长一些, 因此肽键的还原速度 也就慢一些。此外, 霍夫曼降解反应只有当酰胺的氮原子上连有两个氢原子才能发生, 因此酰胺键 $\left(-\mathrm{CONH}_{2}\right)$ 可以发生霍夫曼降解而肽键 $(-\mathrm{CONH}-)$ 不能发生; 倘若能观测到霍夫曼降解的产物, 则 可以作为分子内存在酰胺键 $\left(-\mathrm{CONH}_{2}\right)$ 更加直接的证据, 更加确丵地证明万古霉素中含有天冬酰 胺(含 1 个 $-\mathrm{CONH}_{2}$ 基团), 而不是像谢尔德里克之前猜测的含有异天冬酰胺残基(不含 $-\mathrm{CONH}_{2}$ 基 团) ${ }^{[23]}$ 。

这两个实验都给了康斯坦斯·哈里斯预想之中的结果。他的猜想是对的。基于这些事实, 他提出 了万古霉素的结构 ${ }^{[24]}$, 如图1所示。

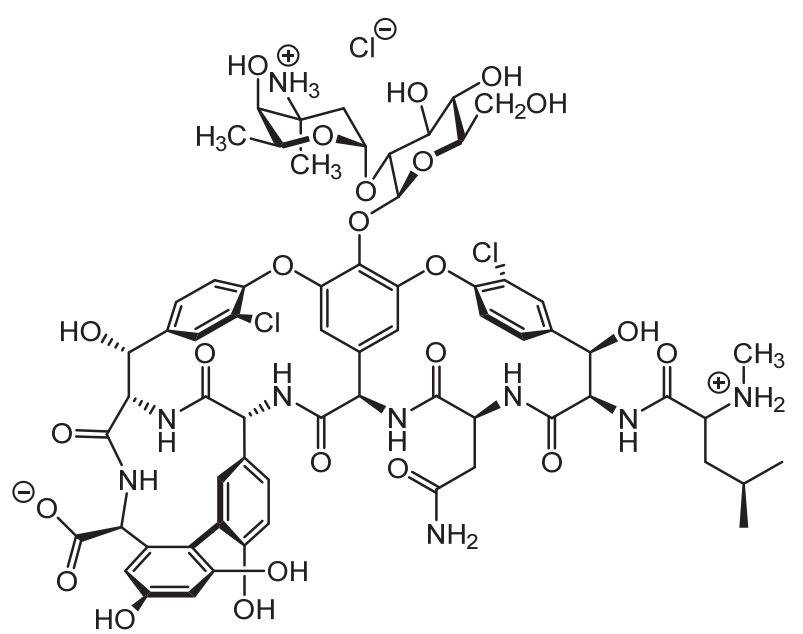

图1 万古霉素的结构

随着对结晶条件的进一步摸索, 人们得到了纯度更高的单晶, 后续的X射线衍射实验也进一步 验证了康斯坦斯·哈里斯所提出的结构 ${ }^{[25,26]}$ 。人们同时发现, 万古霉素中除了氨基酸的碳中心手性之 外, 将肽链交联起来的芳环还存在轴手性和大环阻转面手性这两种手性类型 [27], 并随即通过单晶衍 射确定了手性轴的绝对构型。根据这一系列结果, 人们建立了万古霉素的的三维分子模型, 完成了 从一团迷雾, 到扁平图像, 再到三维具象的结构建立。自此, 万古霉素终于成了一个有血有肉的分 子个体。

而三维结构的建立也使得万古霉素的杀菌机理清晰了起来 ${ }^{[28]}$ 。人们惊奇地发现, 万古霉素恰好 能够像把 “钥匙” 那样, 通过几对氢键 ${ }^{[29]}$, 将细菌细胞中以 L-赖氨酰-D-丙氨酰-D-丙氨酸封端的肽 聚糖 ${ }^{[30]}$ 这把 “锁” 紧密地结合在一起, 形成了稳定的万古霉素-肽聚糖复合物。而当 “锁” 插入 “钥 匙” 中后, 原先肽聚糖 “锁” 与 “锁” 之间的进一步交联被抑制了, 让细菌失去了合成细胞壁的能 
力。为了验证这个作用模型, 研究万古霉素的专家威廉姆斯和他的同事一起, 在核磁共振氢谱上找

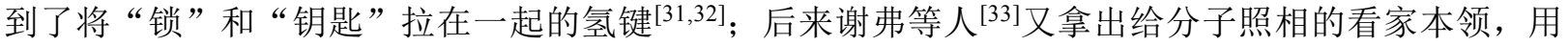
$\mathrm{X}$ 射线拍到了连接着万古霉素和肽聚糖的氢原子。

\section{5 化身千百}

“小万，小万，快醒醒，你又多了一个弟弟！”自从结构确定、灭菌作用模型确定以来，万古 霉素和他们家族的分子都好像凭空有了灵魂, 针对前一代药物暴露出的耐药性、作用模式、副作用 等弊端, 涌现出一批以小万为骨架的新成员 ${ }^{[34]}$ 。只不过因为耐万古霉素细菌的缓慢增加, 小万在临 床一线抛头露面的机会少了些, 也就常常无所事事, 陷入沉睡。

这会儿, 小万的家族又壮大了, 兄弟姐们正在叫他起床呢。小万睁开眼一看, 围在他周围的除 了替考拉宁、达巴万星、奥利万星之外, 又多了一个陌生面孔。他们告诉小万, 这个新成员叫做特 拉万星, 他除了能够与肽聚糖前体的末端结合之外, 同时还能改变细胞膜的通透性, 两大效应协同 作用，杀死革兰氏阳性菌 ${ }^{[35]}$ 。

小万有些惊讶。这些年里, 他每一次醒来, 看到的新成员都会让他感慨药物发展的速度之快。 不知道下一次醒来，身边又会多出哪个小伙伴呢?

“大概还会有效果更强、毒副作用更弱的的弟弟妹妹吧。”想着想着，小万又睡了过去……

\section{参 考 文 献}

[1] 刘否, 许家喜. 大学化学, 2010, 25 (S1), 31.

[2] Hawley, H. B.; Gump, D. W. Am. J. Dis. Child. 1973, 126, 261.

[3] 俞汝勤. 大学化学, 2010,25(S1), 2.

[4] Michal, G.; Schomburg, D. Biochemical Pathways: An Atlas of Biochemistry and Molecular Biology, 2nd ed.; John Wiley \& Sons, Inc.: Hoboken, America, 2012; pp. 14-15.

[5] 杨秀山. 微生物学通报, 1982, 9 (2), 43.

[6] Levine, J. F. Med. Clin. North Am. 1987, 71, 1135.

[7] McCormick, M. H.; Stark, W. M.; Pittenger, G. E.; Pittenger, R. C.; McGuire, J. M. Antibiot. Annu. 1955, 56, 606.

[8] Ahmed, M. O.; Baptiste, K. E. Microb. Drug Resist. 2018, 24, 590.

[9] Diaz, R.; Afreixo, V.; Ramalheira, E.; Rodrigues, C.; Gago, B. Clin. Microbiol. Infec. 2018, $24,97$.

[10] Lechevalier, M. P.; Prauser, H.; Labeda, D. P.; Ruan, J.-S. Int. J. Sys. Bacteriol. 1986, $36,29$.

[11] Panday, P. N.; Sturkenboom, M. Clin. Infect. Dis. 2009, 49, 1964.

[12] Williams, D. H. Nat. Prod. Rep. 1996, 13, 469.

[13] 董朕, 陈晨, 李冰, 周绪正, 张继瑜. 畜牧兽医学报, 2020, 51 (7), 1488.

[14] Fujioka, K; Takeuchi, S.; Tayama, T.; Takei, M.; Ono, A.; Shono, M.; Shichijo, K.; Narita, T.; Kondo, S. IDCases 2021, 24, e01158.

[15] Appel, G. B.; Neu, H. C. N. Engl. J. Med. 1977, 296, 722.

[16] Rothenberg, H. J. JAMA 1959, 171, 1101.

[17] Cook, F. V.; Farrar, W.E. Ann. Intern. Med. 1978, $88,813$.

[18] Hiramatsu, K. Drug Resist. Updates 1998, 1, 135.

[19] Sheldrick, G. M.; Jones, P. G.; Kennard, O.; Williams, D. H.; Smith, G. A. Nature 1978, $271,223$.

[20] Marshall, F. J. J. Med. Chem. 1965, 8, 18.

[21] Williams, D. H.; Kalman, J. R. J. Am. Chem. Soc. 1977, 99, 2768.

[22] Williamson, M. P.; Williams, D. H. J. Am. Chem. Soc. 1981, 103, 6580.

[23] Harris, C. M.; Harris, T. M. J. Am. Chem. Soc. 1982, 104, 4293. 
[24] Harris, C. M.; Kopecka, H.; Harris, T. M. J. Am. Chem. Soc. 1983, 105, 6915.

[25] Merkel, A. B.; Temple, G. K.; Burkart, M. D.; Losey, H. C.; Beis, K.; Walsh, C. T.; Naismith J. H. Acta Crystallogr., Sect D: Biol. Crystallogr. 2002, 58,1226

[26] Schäfer, M.; Pohl, E.; Schmidt-Bäse, K.; Sheldrick, G. M.; Hermann, R.; Malabarba, A.; Nebuloni, M.; Pelizzi, G. Helv. Chim. Acta 1996, 79, 1916.

[27] 盛思敏, 沈珍, 陈建成. 大学化学, 2020, 35 (11), 90 .

[28] Williams, D. H.; Bardsley, B. Angew. Chem. Int. Ed. 1999, 38, 1172.

[29] 王毓江, 唐黎明. 化学进展, 2006, $18(\mathrm{Z1}), 308$.

[30] Perkins, H. R. Biochem. J. 1969, 111, 195.

[31] Williams, D. H. Acc. Chem. Res. 1984, 17 (10), 364.

[32] Williams, D. H.; Williamson, M. P.; Butcher, D. W.; Hammond, S. J. J. Am. Chem. Soc. 1983, 105, 1332.

[33] Schäfer, M.; Schneider, T. R.; Sheldrick, G. M. Structure 1996, 4, 1509.

[34] Nicolaou, K. C.; Boddy, C. N. C.; Bräse, S.; Winssinger, N. Angew. Chem. Int. Ed. 1999, 38, 2096.

[35] Lunde, C. S.; Hartouni, S. R.; Janc, J. W.; Mammen, M.; Humphrey, P. P.; Benton, B. M. Antimicrob. Agents Chemother. $2009,53,3375$. 